\title{
Multiscale hydro-thermo-chemo-mechanical coupling: Application to alkali-silica reaction
}

\author{
T. $\mathbf{W u}^{\mathrm{a}, *}$, İ. Temizer $^{\mathrm{b}}$, P. Wriggers ${ }^{\mathrm{a}}$ \\ a Institute of Continuum Mechanics, Leibniz University of Hannover, Appelstr. 11, 30167 Hannover, Germany \\ ${ }^{\mathrm{b}}$ Department of Mechanical Engineering, Bilkent University, 06800 Ankara, Turkey
}

\section{A R T I C L E I N F O}

\section{Article history:}

Received 7 August 2013

Received in revised form 3 December 2013

Accepted 13 December 2013

Available online 11 January 2014

\section{Keywords:}

Multiscale

Alkali-silica reaction

Concrete

Cement paste

Homogenization

\begin{abstract}
A B S T R A C T
Alkali-Silica Reaction (ASR) is a complex chemical process that affects concrete structures and so far various mechanisms to account for the reaction at the material level have already been proposed. The present work adopts a simple mechanism, in which the reaction takes place at the micropores of concrete, with the aim of establishing a multiscale framework to analyze the ASR induced failure in the concrete. For this purpose, 3D micro-CT scans of hardened cement paste (HCP) and aggregates with a random distribution embedded in a homogenized cement paste matrix represent, respectively, the microscale and mesoscale of concrete. The analysis of the deterioration induced by ASR with the extent of the chemical reaction is initialized at the microscale of $\mathrm{HCP}$. The temperature and the relative humidity influence the chemical extent. The correlation between the effective damage due to ASR and the chemical extent is obtained through a computational homogenization approach, enabling to build the bridge between microscale damage and macroscale failure. A 3D hydro-thermo-chemo-mechanical model based on a staggered method is developed at the mesoscale of concrete, which is able to reflect the deterioration at the microscale due to ASR.
\end{abstract}

(c) 2013 Elsevier B.V. All rights reserved.

\section{Introduction}

\subsection{Concrete}

Good durability to cost ratio renders concrete one of the most widely used construction materials. However, it is an extremely complex heterogeneous material with a random microstructure at different length scales. At the macroscale, it is treated as a homogeneous material. One scale lower, the mesoscale, includes a binding matrix, aggregates and pores with broad size distributions as well as interfacial zones between the aggregates and the matrix. Finally, the microscale constitutes the finest structural scale and is represented by the microstructure of hardened cement paste (HCP), which is comprised of hydration products, unhydrated residual clinker and micropores [1]. The observable or macroscale failure of the concrete caused by various environmental attacks, such as frost [2] and Alkali-Silica Reaction (ASR) [3] as well as severely mechanical loading [4], can be explained by the variation of the underlying microstructure of the concrete. Therefore, it is desirable that a correlation between the macroscopic failure and the variation of the microstructure is established. However, the high cost of the conventional experiment in the laboratory high-

\footnotetext{
* Corresponding author. Tel.: +49 (511) 7624118.

E-mail address: wu@ikm.uni-hannover.de (T. Wu).
}

lights the need for an alternative approach. With the continuous improvement of computational power and the development of non-destructive evaluation techniques such as computer tomography, one can obtain the microstructure of the material at the microscale and carry out the analysis numerically.

Over the recent years, multiscale models were developed to analyze the failure in the concrete suffering from various attacks. Hain and Wriggers [2] have evaluated the damage due to frost in HCP using a finite element model that is based on the threedimensional computer-tomography scans of HCP, and the obtained effective damage was upscaled to the next scale of the concrete via a computational homogenization approach. Cusatis et al. [5] developed an equivalent macroscopic cohesive law reflecting the mesolevel failure mechanisms, where the investigation of the fracture behavior at the lower scale was carried out through a lattice-type model. A coupled macro-meso-micro model was described by Nguyen et al. [1] to link the micro-diffusive damage and the macro-crack in the concrete, by incorporating a cohesive zone model (CZM) within an iterative $\mathrm{FE}^{2}$ approach. Eckardt and Könke [6] adopted the nonlocal damage model to present the initiation, propagation and coalescence of microcracks at the mesoscale and subsequent formation of a macroscopic crack. In addition, the adaptive domain decomposition method was used to analyze the multiscale failure in the concrete. Ghosh and Chaudhuri [7] developed a multiscale failure model for concrete, where the 
material far from the fracture process zone was described by the homogenized elastic model, while a nonlinear model was used in the fracture process zone. The fracture at the mesoscale was modeled by CZM in the context of an enriched partition of unity meshfree method. Idiart et al. [8] focused on the external sulfate attack on the concrete specimen at the meso-level, where zero-thickness interface elements with fracture-based constitutive laws were adopted. Moreover, the influence of discrete cracks on the transport of ions was explicitly taken into account. While these works successfully capture various features of failure mechanisms, the goal of the present work is to establish a direct link between concrete failure and the microscale driving mechanisms.

\subsection{Alkali-silica reaction}

ASR, discovered in the 1940s, is a long-term chemical reaction and detrimental to a concrete structure. ASR is characterized by the breakdown of the siloxane bonds $(\mathrm{Si}-\mathrm{O})$ in poorly crystallized silica of aggregates, which is attacked by the hydroxyl ions $\mathrm{OH}^{-}$ from the pore solution in the HCP $[3,9,10]$. It leads to the formation of an alkali-silica gel, which swells in the presence of water and exerts pressure on the surrounding material. It induces microand, eventually, macro-cracking of the structure when the tensile strength of concrete is exceeded $[3,9,10]$. Overall, three factors are indispensable for ASR:

- alkali content in the pore solution,

- poorly crystallized silica in aggregates,

- moisture content.

ASR is a complex phenomenon and it is still a challenging topic from the multiscale modeling point of view due to different manifestations of ASR at the material level of the concrete. A micromechanical approach was proposed by Lemarchand et al. [9], where the topochemical and through-solution mechanisms were accounted for. The gel was generated at sites of silica dissolution, referred to as topochemical mechanism, and the gel was produced in the porous space, referred to as through-solution mechanism. McGowan and Vivian [11] have elaborated that a solid layer was formed on the surface of the aggregate to absorb the moisture from the pore solution in HCP, and then the solid substance was transferred to the gel. Some authors [12-14] proposed that the aggregate was tightly packed with an insoluble rim, allowing the penetration of the alkaline solution, rather than the viscous alkali-silica gel. Multon et al. [15] predicted that the gel was generated inside the aggregates after alkali and hydroxyl ions diffuse into the aggregates to break the silanol bonds. Then the gel permeates through the connected porous volume between aggregates and cement paste. Therefore, the gel was maintained inside aggregates and distributed the expansive pressure to aggregates. Meanwhile, Dunant et al. and Haha et al. [16,17] highlighted the predominant effect of the gel formation in the aggregates, leading to damage in the aggregates themselves and to subsequently damage the surrounding cement paste. Experiments by Ponce and Batic [18] indicated that the different location of the gel from mechanisms reported in [15-17] can be explained by the types of aggregates. The experiment [18] captured that cracking patterns of ASR-affected concrete rely on the mineralogical nature of the aggregates through petrographic examination with a stereobinocular and a polarizing microscope. It was also observed that aggregates such as opal and vitreous volcanic rocks result in the formation of the gel at the interface between aggregate and cement paste, thus causing the cracking in cement paste. On the other hand, mixed mineralogy aggregates formed cracks in both the aggregates and cement paste.

\subsection{Review of numerical models on alkali-silica reaction}

During the last decade, several numerical models have been developed to predict the ASR induced failure at different length scales of the concrete. Huang and Pietruszczak [28] established the correlation between the expansion strain due to ASR and the mechanical degradation at the macroscale of the concrete, where the expansion strain had a similar formulation with the thermal dilation strain. An advanced thermo-chemo-mechanical model was developed by Ulm et al. [3] in the framework of Biot's theory, where the concrete was conceived as a two-phase material including the expansive gel and the homogenized concrete skeleton. Moreover, the volumetric expansion of the gel was evaluated as a function of the reaction kinetics, which is influenced by the temperature. Comi et al. [31] developed the model based on [3], where not only temperature but also relative humidity contribute to the extent of the reaction. In addition, the investigation on ASR at the macroscale of the concrete was developed by Bangert et al. [30], where the concrete was treated as a mixture of three superimposed constituents through the theory of porous media: skeleton, pore liquid and pore gas. The model was based on converting the mass of unreacted material into the mass of the reacted material in the skeleton. Various analytical models based on empirical equations were also developed to explain ASR at the mesoscale of the concrete, see [10,15]. For instance, Bažant and Steffens [10] proposed that the chemical reaction kinetics was related to the diffusion process of the reactants, leading to the subsequent fracture in the characteristic unit cell of the concrete modeled with one spherical glass particle. Detailed numerical models at the mesoscale were limited. Comby-Peyrot et al. [37] introduced a three-dimensional mesoscopic model, where aggregates are randomly distributed in the cement matrix. The damage in the cement matrix was caused by the isotropic dilatation phenomenon in the reactive aggregates induced by ASR. Dunant and Scrivener [16,34] proposed a 2D finite element/extended finite element framework to qualitatively depict the ASR induced deterioration at the mesoscale of the concrete. The growing gel pockets defined in the aggregates trigger the damage to them, where the geometry of gel swelling is represented by updating the enrichment function. Alnaggar et al. [38] adopted the framework of the Lattice Discrete Particle Model (LDPM) to capture the ASR induced crack patterns at the mesoscale of the concrete. The expansion of the gel occurs at the level of each individual aggregate particle. Due to lack of reliable microscale models and representations, no results were contributed at the microscale of the concrete with the application to ASR. The general review of numerical models on ASR is listed in Table 1. For an overview of ASR with detailed references, see $[17,39]$.

\subsection{Scope of the present work}

ASR is a complex chemical reaction and its mechanisms at the material level are still in dispute. Some numerical models concerning ASR have already been successfully set up at the macro- and meso-scale of the concrete. The objective of the present work is to establish a multiscale computational framework for predicting ASR induced damage where, for the first time to the best knowledge of the authors'. The analysis on ASR is carried out at the microscale of the concrete and the ASR induced deterioration is upscaled to the mesoscale. The general scope of the study is as follows:

- to investigate the contribution of the chemical extent of ASR to the expansion strain of the gel at the micropore and the resulting deterioration of HCP, 
Table 1

General review of numerical models of ASR.

\begin{tabular}{|c|c|c|c|}
\hline $\begin{array}{l}\text { Length } \\
\text { scale }\end{array}$ & Multiphysics & References & General description \\
\hline Macroscale & Mechanical & [19-23] & Extensive work has been carried out to identify the expansion strain as a function of the stress tensor \\
\hline Macroscale & Chemo-mechanical & $26,15,27]$ & $\begin{array}{l}\text { A nonlinear relation between the expansion strain of concrete and the chemical extent was adopted to construct a } \\
\text { model at the structural level }\end{array}$ \\
\hline Macroscale & $\begin{array}{l}\text { Hydro-thermo- } \\
\text { chemo-mechanical }\end{array}$ & {$[3,28-32]$} & $\begin{array}{l}\text { Temperature and relative humidity contributed to the chemical extent of ASR and the expansion strain, leading to } \\
\text { the failure of the concrete structure }\end{array}$ \\
\hline Mesoscale & Chemo-mechanical & $\begin{array}{l}{[16,33-} \\
37]\end{array}$ & $\begin{array}{l}\text { Randomly distributed aggregates were embedded in the matrix to represent the mesoscale of the concrete. The } \\
\text { formation and expansion of ASR gel are described at the level of each individual aggregate. The influences of } \\
\text { aggregates on the expansion strain and the subsequent damage in the concrete were taken into account }\end{array}$ \\
\hline Microscale & $\begin{array}{l}\text { Hydro-thermo- } \\
\text { chemo-mechanical }\end{array}$ & $\begin{array}{l}\text { Current } \\
\text { work }\end{array}$ & $\begin{array}{l}\text { It initializes the analysis of ASR at the microscale of } \mathrm{HCP} \text { and investigates the correlation between damage due to } \\
\text { ASR and chemical extent. The extent is influenced by the temperature and relative humidity. The ASR induced } \\
\text { damage at the microscale is upscaled to the mesoscale via computational homogenization towards observable } \\
\text { failure }\end{array}$ \\
\hline
\end{tabular}

- to obtain the correlation between the effective mesoscale damage due to ASR and the chemical extent through computational homogenization,

- to carry out hydro-thermo-chemo-mechanical coupling based on a staggered method at the mesoscale, where transient temperature and relative humidity are employed, thereby establishing a multiscale and multiphysics model to predict the failure due to ASR.

The structure of the paper is as follows. Section 2 will introduce the chemical mechanism of ASR as well as establish the chemical extent as a function of the temperature and the relative humidity. The representation of $\mathrm{HCP}$ and the constitutive equations of its components are explained in Section 3, where computational homogenization approach will be highlighted, building the link between the microscale and the macroscale. Section 4 will discuss details of generating the mesoscale representation of concrete. A two-step homogenization approach will be introduced to seek the expansion coefficient of the gel at the microscale in Section 5. Section 6 will present the hydro-thermo-chemo-mechanical framework based on a staggered method at the mesoscale. The last section concludes with a brief summary and an outlook.

\section{Kinetics of alkali-silica reaction}

ASR can be specifically modeled as a two-stage process, involving the dissolution of the silica and the swelling of the gel. The work [40] proposed that the dissolution stage can be described by a first-order kinetic law, which was widely adopted - see $[3,29,31,41]$. In addition, Larive [41] verified the dependence of the chemical reaction kinetics on the temperature and the relative humidity.

\subsection{Chemical reaction kinetics}

The aforementioned first-order kinetic law can be expressed through

$\tilde{t} \frac{d \xi}{d t}=1-\xi$

where $t$ is the time, $\tilde{t}$ is the intrinsic time of the reaction and $\xi \in[0,1]$ is the chemical extent, measuring the progression of the reaction: $\xi=0$ is the beginning and $\xi=1$ is the end. In view of the macroscopic experiment, $\xi$ is defined by the ratio between the current expansion strain and the terminal one of the concrete specimen. The explicit equation of $\tilde{t}$ is proposed in [41]

$\tilde{t}=\tau_{c h} \frac{1+\exp \left[-\tau_{\text {lat }} / \tau_{c h}\right]}{\xi+\exp \left[-\tau_{\text {lat }} / \tau_{c h}\right]}$ where $\tau_{\text {lat }}$ is the latency time and $\tau_{c h}$ is the characteristic time, respectively corresponding to the initiation and the development period of ASR from a practical point of view. Larive [41] addressed that $\tau_{\text {lat }}$ and $\tau_{c h}$ depend on the temperature and the relative humidity. If only the temperature factor is taken into account, the dependence was proposed in the form

$\tau_{\text {lat }}(T)=\tau_{\text {lat }}(\bar{T}) \exp \left[U_{\text {lat }}(1 / T-1 / \bar{T})\right]$

and

$\tau_{c h}(T)=\tau_{c h}(\bar{T}) \exp \left[U_{c h}(1 / T-1 / \bar{T})\right]$

where $T$ is the temperature, $\bar{T}=38{ }^{\circ} \mathrm{C}$ is the reference temperature and, $U_{\text {lat }}$ and $U_{\text {th }}$ are the Arrhenius activation energies $\left(U_{c h}=5400 \pm 500 \mathrm{~K}\right.$ and $U_{\text {lat }}=9400 \pm 500 \mathrm{~K}$ [3]). The explicit equation of the chemical extent $\xi$ only considering isothermal case is obtained by taking the integral of Eq. (2.1):

$\xi(t, T)=\frac{1-\exp \left[-t / \tau_{c h}(T)\right]}{1+\exp \left[-t / \tau_{c h}(T)+\tau_{\text {lat }}(T) / \tau_{c h}(T)\right]}$

In Fig. 1, the S-shaped curve illustrates the chemical extent under constant temperature $T=25^{\circ} \mathrm{C}$ with respect to days, where the regions and physical meanings of the latency time and the characteristic time are indicated. Overall, the S-shaped curve represents three stages of ASR [9]: initiation, reaction, and exhaustion.

\subsection{Influence of temperature on chemical extent}

It is well understood that ASR is activated by the temperature, according to either the Arrhenius law or the experiment from La-

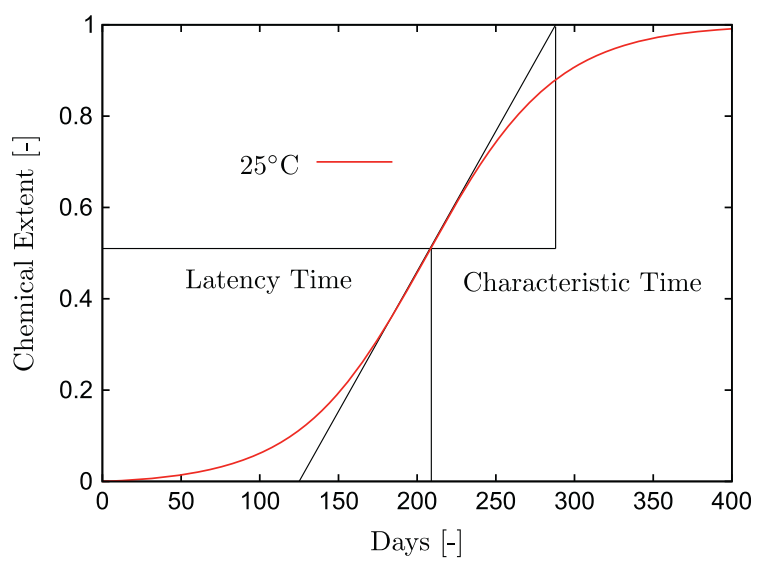

Fig. 1. Latency time and characteristic time in the S-shape curve of chemical extent under constant temperature. 
rive [41], so that the higher the temperature, the faster the reaction is. Studies associated with the influence of the temperature on the chemical extent $\xi$ and the subsequent deterioration in the concrete have been reported in the literature, see $[3,29]$. Clearly, the chemical extent $\xi$ as an irreversible internal variable can be calculated based on various temperature history inputs. Fig. 2 present examples of temperature variations with time and the calculated chemical extent.

\subsection{Influence of relative humidity on chemical extent}

The influence of the relative humidity on ASR is another significant concern $[42,43]$. Here, there are two main functions of water:

- transport medium of the ionic species,

- reactant in the swelling of the amorphous gel.

Kurihara and Katawaki [42] and Tomosawa et al. [44] have indicated that ASR can be inhibited if the relative humidity in the concrete is below a certain threshold. Nilsson [43] explained that there is no ASR induced degradation when the relative humidity drops below 0.8 . Ludwig [45] stated that the threshold quantity of the relative humidity to initialize ASR is between 0.8 and 0.85 under a constant temperature of $20^{\circ} \mathrm{C}$. Different threshold values found in the literature can be explained by different experimental conditions and mixtures of the concrete. The experimental data from [41] introduced the dependence of the latency time and the characteristic time on the relative humidity, see Fig. 3, where the threshold value of relative humidity to activate the reaction is around 0.6. This observation is adopted in this contribution. The work of Larive [41] provided the experimental data of the latency time and the characteristic time with respect to saturation degree. Due to mechanisms of molecular absorption/desorption, capillary condensation, surface tension and disjoining pressure, a nonlinear relationship results between the saturation degree and the relative humidity, which is complexly affected by the temperature, see [46]. In this work, the effects of absorption and desorption are not taken into account, accordingly, saturation degree is ideally replaced by relative humidity. The discrete sampling data with respect to relative humidity from [41] can be approximated by the continuous curves through

$$
\begin{aligned}
\tau_{i}(T, S) & =\left(\tau_{i}(\bar{T}, 1)+\frac{\tau_{i}(\bar{T}, 0)-\tau_{i}(\bar{T}, 1)}{1+a_{1, i} \exp \left[-\frac{a_{2, i}(1-2 S)}{S(1-S)}\right]}\right) \exp \left[U_{i}\left(\frac{1}{T}-\frac{1}{\bar{T}}\right)\right], \quad i \\
& =\text { ch, lat }
\end{aligned}
$$

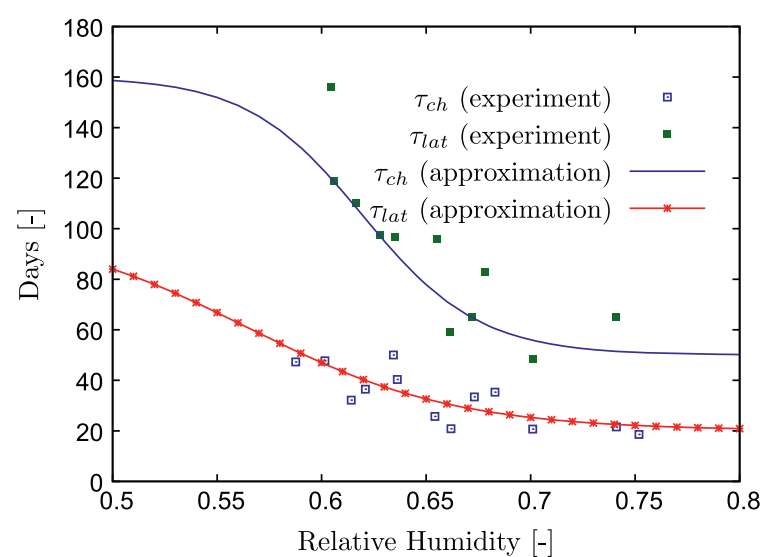

Fig. 3. Experimental data from [41] and their approximations to take into account the effect of the relative humidity on the latency time and the characteristic time.

where $S$ is the relative humidity and lower indicates $i=($ ch, lat $)$ describe the latency time and the characteristic time, respectively. In addition, 0 and 1 in the dependence of $\tau_{i}$ represent the beginning and the end of the chemical reaction. The dependence of the chemical extent on the moisture content assumed in Eq. (2.6) was first proposed in [31]. The coefficients of the approximations are listed in Table 2 and the corresponding curves are provided in Fig. 3.

The approximate curves in Fig. 3 can be transferred into the expression of the chemical extent $\xi$ in Eq. (2.5), so that the effect of the relative humidity on ASR can be incorporated into the present framework. In order to capture the irreversible characteristics of ASR while taking into account the effect of relative humidity, Eq. (2.5) and (2.6) are utilized to calculate the chemical extent $\xi$, where relative humidity contributes to the latency time and the characteristic time through Eq. (2.6). Subsequently, the obtained time quantities are adopted to calculate the chemical extent $\xi$ through Eq. (2.5). Examples of relative humidity histories with respect to days and the corresponding results for the chemical extent $\xi$ are illustrated Fig. 4 . The influences of the temperature and the

Table 2

The coefficients of the approximations to take into account the effect of the relative humidity on the latency time and the characteristic time.

\begin{tabular}{lllll}
\hline$i$ & $\tau(\bar{T}, 0)$ (Days) & $\tau(\bar{T}, 1)$ (Days) & $\mathrm{a} 1$ & $\mathrm{a} 2$ \\
\hline$t_{\text {lat }}$ & 160 & 50 & 20 & -18.5222 \\
$t_{c h}$ & 100 & 20 & 1 & -10.52 \\
\hline
\end{tabular}

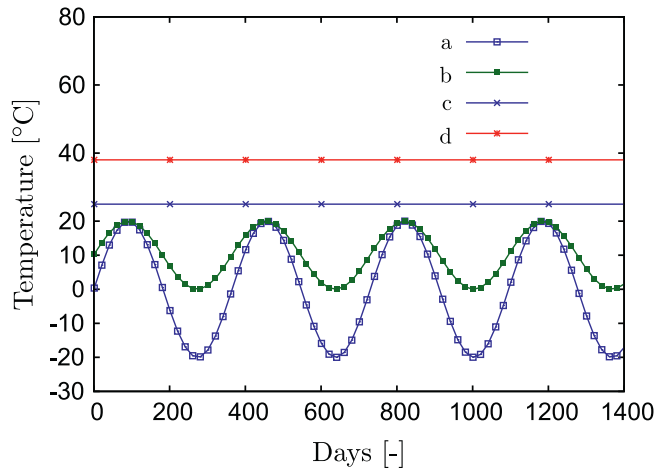

(a)

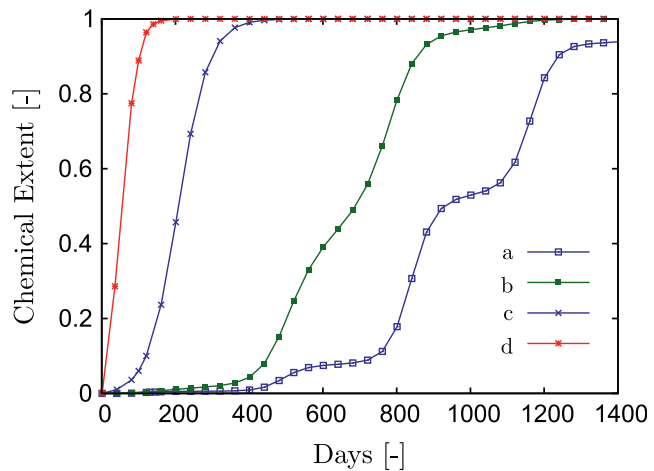

(b)

Fig. 2. (a) Various temperature histories with respect to days and (b) the calculated chemical extent history under the imposed temperature variation. 
relative humidity on the chemical extent $\xi$ have so far been investigated separately. In order to involve both influences simultaneously, the combination of Eqs. (2.3), (2.4) and (2.6) are employed, see Fig. 5, where the latency time and the characteristic time are functions of the temperature and the relative humidity. This establishes the basis of the hydro-chemo-thermal coupling in the present work.

\subsection{Fundamentals and assumptions}

In the laboratory, the reaction is often accelerated by enforcing high temperature and large relative humidity, yet is still relatively slow. In Section 2.1, the obtained S-shape curve originating from Eq. (2.5) captures two important stages of ASR: the initiation and the development period $[9,45]$. The influences of the temperature and the relative humidity on the chemical extent are analyzed in Section 2.2 and 2.3. It is noted that the observation that higher temperature and larger relative humidity cause larger expansion strain of the concrete specimen induced by ASR can be found in $[9,45]$, but this issue is not taken into account in this work. Previous work on the mechanisms of ASR was presented in Section 1.3. It is recalled that the ASR mechanism is not fully resolved. The objective of the present work is to initiate the investigation of the deterioration due to ASR at the microscale of HCP, and then to build the link between the microscale and the mesoscale of the concrete. Before moving further, some significant assumptions and simplifications need to be addressed:
- Despite the presence of several proposes regarding ASR are according to the experimental observation, the present work concentrates on the through-solution mechanism [9]: gels are produced in micropores of HCP, and they exert pressure uniformly on the surrounding material.

- Gel rapidly fills up the micropore of HCP once ASR starts, yet exerting no pressure on the surrounding material at the beginning. In other words, the transition from the dissolution stage to the swelling stage is not modeled.

- The expansion strain of the gel at the microscale is defined by the product of the expansion coefficient of the gel and chemical extent $\xi$. In addition, the expansion coefficient of the gel is assumed to remain permanent during the chemical reaction: it is independent of the temperature, relative humidity, time and position.

- The expansion induced by ASR at the micro and macroscale is assumed to be isotropic.

- The influences of reactivity types and size of aggregates are not taken into account [16,17].

- Scalar multiscale variables are typically projected as constants from the upper to the lower scales $[47,48]$. Complying with this observation, temperature and humidity mediated chemical extent variable is projected uniformly into the micropores of representative volume element (RVE) at the microscale of HCP. Hence, the analysis of the microscale does not require a full numerical coupling among all fields.

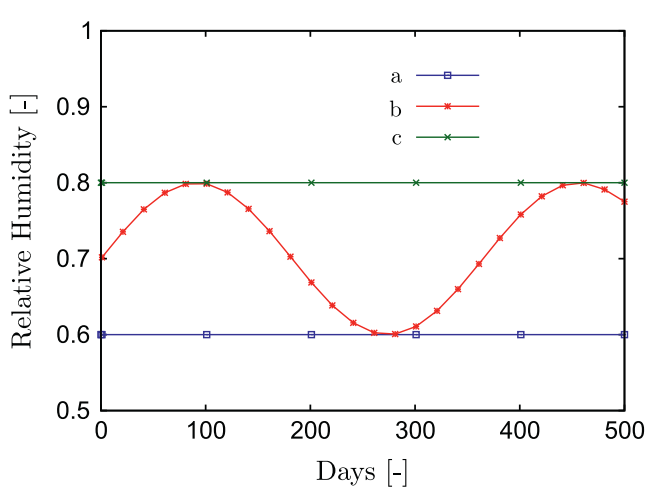

(a)

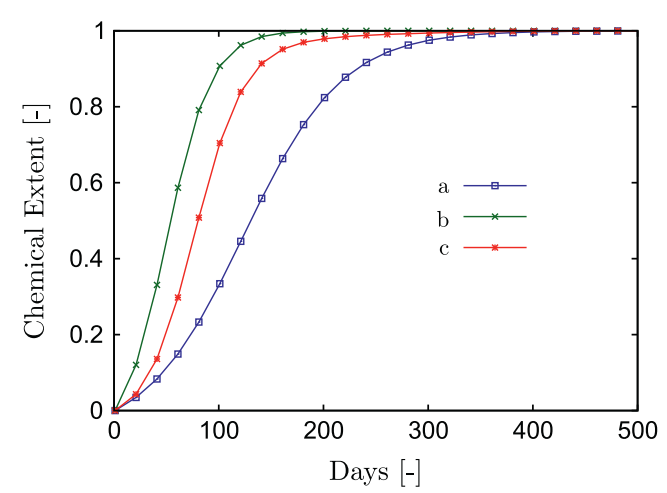

(b)

Fig. 4. (a) Various relative humidity inputs with respect to days and (b) the corresponding chemical extent evolutions.

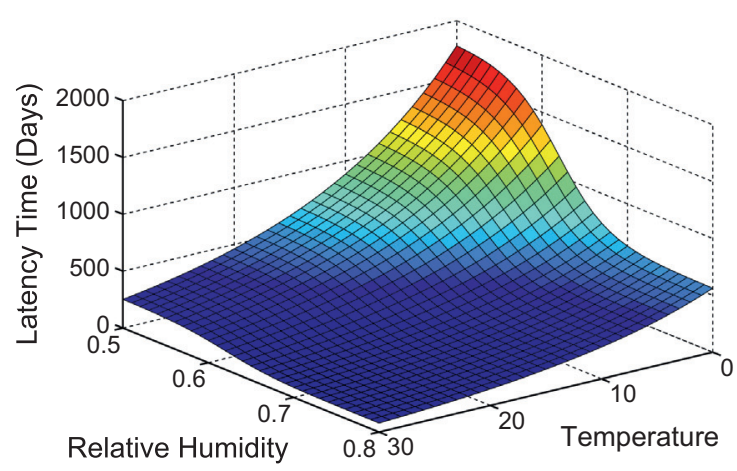

(a)

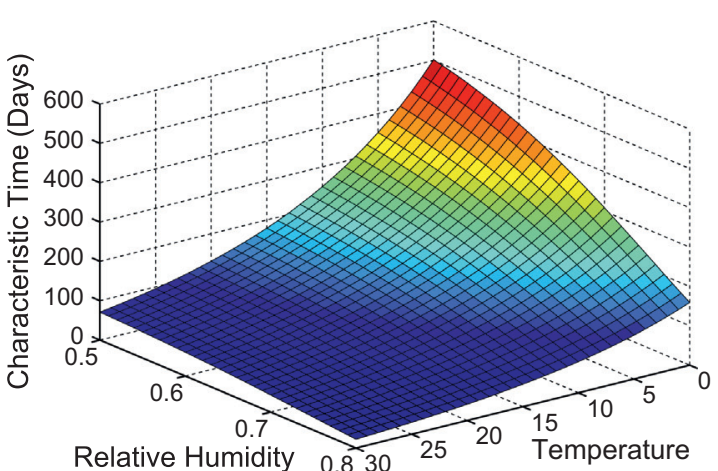

(b)

Fig. 5. (a) The latency time and (b) the characteristic time as functions of the temperature and the relative humidity. 
Based on these, a coupled hydro-thermo-chemo-mechanical simulation framework for the mesoscale of concrete will be developed in the remaining sections. For this purpose, each scale of concrete is treated next in an interactive fashion.

\section{Microscale of concrete}

\subsection{Representation of hardened cement paste}

HCP as the microscale of the concrete is comprised of the hydration product, the unhydrated residual klinker and micropores. Computer Tomography (CT) is a non-destructive evaluation technique for producing 3D images of a specimen through X-ray, enabling to obtain the microstructure of the material and subsequently analyze the mechanical behavior in a numerical manner. In this work, a cement specimen with a length of $1750 \mu \mathrm{m}$ was used to obtain the microstructural geometry of HCP through three-dimensional micro-CT scans with a resolution of $1 \mu \mathrm{m}$ per voxel dimension. Due to this underlying voxel data structure, the natural element to use within the finite element method to discretize the microstructure is an 8-node brick where each element is assigned to a single material phase. This choice allows a straightforward transition from the micro-CT scan data to the numerical analysis stage and is realized based on earlier work $[2,46]$. Three- and two-dimensional representations of HCP are displayed in Fig. 6, where the pale green parts are hydration products, the blue parts are the micropores and orange parts are the unhydrated residual clicker. The volume fraction of the hydration products is $84 \%$, the one of micropores is $14 \%$ and the one of the unhydrated portion is $2 \%$. Obviously, hydration products have the dominant volume fraction in HCP, although the micropore phase with a lower volume fraction plays a significant role in the performance of HCP.

\subsection{Constitutive equation of components in HCP}

Among other continuum damage models which can depict the failure of HCP, the simplified constitutive model developed by Hain and Wriggers $[2,49]$ is employed in this work where damage was constrained to the hydration products because of its high volume fraction in the HCP. Within this simplified model, the mechanical strain $\epsilon^{u}$ is comprised of an elastic $\epsilon^{e l}$ and a crack $\epsilon^{c r}$ strain

$\boldsymbol{\epsilon}^{u}:=\boldsymbol{\epsilon}^{e l}+\boldsymbol{\epsilon}^{c r}$

The stress $\boldsymbol{\sigma}$ is split into the volumetric and deviatoric parts in a classical manner

$\boldsymbol{\sigma}:=C_{v o l} \kappa_{h} \operatorname{tr} \boldsymbol{\epsilon}^{e l} \mathbf{1}+C_{i s o} 2 \mu \operatorname{dev} \boldsymbol{\epsilon}^{e l}$

where $C_{v o l} \in[0 ; 1]$ and $C_{i s o} \in[0 ; 1]$ control the remaining stiffness of the volumetric and the deviatoric parts. $\kappa_{h}$ is the bulk modulus of the hydration product and $\mu$ is the Lamé constant. The occurrence of this damage is induced by the volumetric deformations, such as the expansion of the gel. The update of damage works as follows: if vertvol $\epsilon^{e l} \mid \geqslant \alpha$ at the initial loading stage, $C_{\text {vol }} \approx 0$ and $C_{\text {iso }}=\beta_{\text {iso }}$. When $\mid$ vol $\epsilon^{e l} \mid \leqslant \alpha$ during the unloading process, $C_{\text {vol }}=\beta_{\text {vol }}$ and $C_{\text {iso }}=\beta_{\text {iso }}$. When the material is loaded again and if $\mid$ vol $\epsilon^{e l} \mid \geqslant \gamma \alpha, C_{v o l} \approx 0$ and $C_{i s o}=\beta_{\text {iso }}$. More details of this algorithm can be found in $[2,49]$ from which the damage parameters, determined through experimental results, are adopted: $\left[\alpha, \beta_{i s o}, \beta_{\text {vol }}, \gamma\right]=[0.004,0.05,0.01,0.50]$. The unhydrated residual klinker is assumed to behave elastically. The undamaged mechanical properties of the hydration product and the unhydrated residual klinker in HCP are listed in Table 3.

\subsection{Gel in micropore of $\mathrm{HCP}$}

The deterioration of concrete resulting from ASR is induced by the expansion of the gel. Hence, the mechanical properties of the gel play a central role. The gel has a complex structure that depends on the composition of HCP and on the types of the aggregates. Moreover, due to its unstable chemical characteristics, the experimental determination of the gel properties is challenging. The observation that the gel is chemically similar to calciumsilicate-hydrate ( $\mathrm{C}-\mathrm{S}-\mathrm{H})$ enables it to be conceived as a nearly incompressible material with a Poisson's ratio of 0.49997, see [34].

Standard finite elements cannot appropriately handle incompressibility due to volumetric locking. Higher-order interpolations may suffer less from this problem, but the displacement solution is still of low order accuracy [52-54]. In this work, the classical Q1P0 method is employed where the displacement and pressure are the primary unknowns. The hydrostatic pressure induced by the expansion of the gel in the micropores (see Fig. 7) is expressed through

$P:=\kappa_{g}(\operatorname{tr} \epsilon-A)=\kappa_{g} \operatorname{tr} \epsilon-\kappa_{g} \beta \xi$

where $A=\beta \xi$ denotes the expansion strain of the gel, $\beta$ presents the expansion coefficient of the gel, $\xi$ is the chemical extent and $\kappa_{g}$ is the bulk modulus of the gel. As assumed before, the expansion strain of the gel $A$ is proportional to the chemical extent $\xi$. The mechanical properties of the gel are listed in Table 3. Although $\beta$ is still unknown, an example with $\beta=0.002$ is tested, in order to

Table 3

Mechanical properties of components in HCP.

\begin{tabular}{llll}
\hline Part & $E\left(\mathrm{~N} / \mathrm{mm}^{2}\right)$ & $v(-)$ & $\begin{array}{l}\text { Bulk modulus } \\
\kappa\left(\mathrm{N} / \mathrm{mm}^{2}\right)\end{array}$ \\
\hline $\begin{array}{llll}\text { Unhydrated residual } \\
\text { clinker }\end{array}$ & 132,700 & $0.30[50]$ & - \\
Hydration product & {$[50]$} & & \\
Gel & $24,000[50]$ & $0.24[50]$ & - \\
& - & 0.49997 & $25,000[51]$ \\
& & {$[34]$} & \\
\hline
\end{tabular}

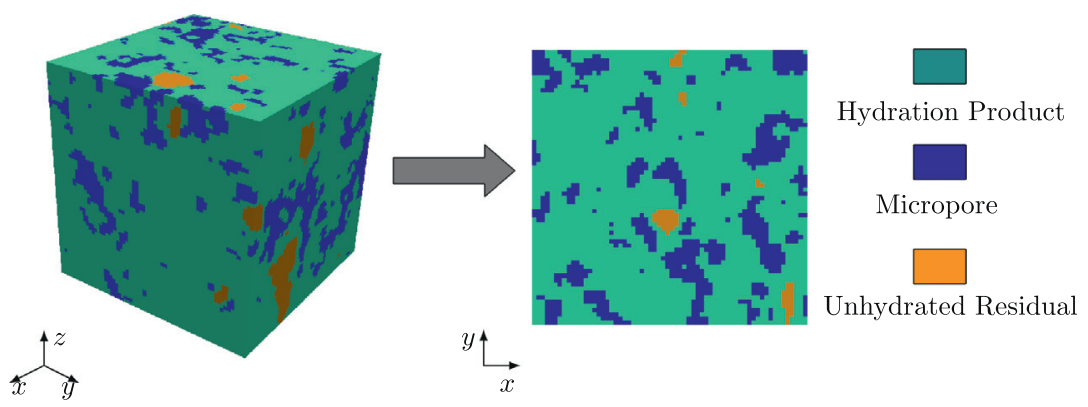

Fig. 6. Three- and two-dimensional representations of HCP extracted from a micro-CT scan. 

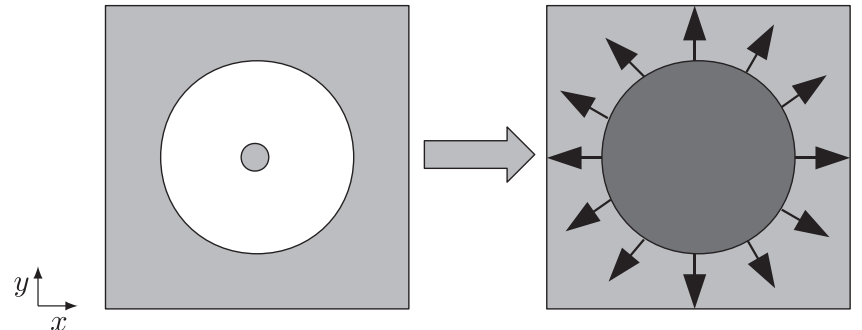

Fig. 7. Swelling of gel to exert uniform pressure to surrounding matrix.

capture the distribution and evolution of the damage in HCP with the extent of ASR, see Fig. 8. It is observed that the damage concentrates in the hydration products in the vicinity of the micropores since it is triggered by the expansion of the gel in a micropore.

\subsection{Homogenization approach to link microscale and mesoscale}

The fundamental methodology of homogenization is to characterize the effective response of the heterogeneous material by approximately identifying and testing an RVE [55]. The choice of the RVE determines the accuracy and the efficiency of computa- tional homogenization. An RVE size of $64 \times 64 \times 64 \mu \mathrm{m}^{3}$ has already been verified to be satisfactory, see $[2,50]$. Based on an RVE, in the elastic regime, the effective response is then quantified by the elasticity tensor $C^{\text {eff }}$

$\langle\boldsymbol{\sigma}\rangle=C^{e f f}:\langle\boldsymbol{\epsilon}\rangle$

where the volume average of a quantity $\langle\bullet\rangle$ is defined by

$\langle\bullet\rangle=\frac{1}{|\Omega|} \int_{\Omega} \bullet d \Omega$

with $\Omega$ as the analysis volume.

Boundary conditions (BCs) prescribed on an RVE are chosen to satisfy Hill's criterion, typically of the linear displacement, uniform traction or periodic type. Due to the presence of different materials on the surface of the sample, pure traction and periodic type BCs may cause locally overly deformed meshes in the computation. Displacement BCs are favorable in this sense, although they can result in the overestimation of the macroscopic property for a given microstructural sample. The combination of the displacement BCs and the window method can overcome the aforementioned problem, essentially by emulating periodic BCs and hence delivering faster convergence to the effective response with increasing sample size during the RVE identification process - see [56] for details. In the context of the window method, the RVE is embedded in a

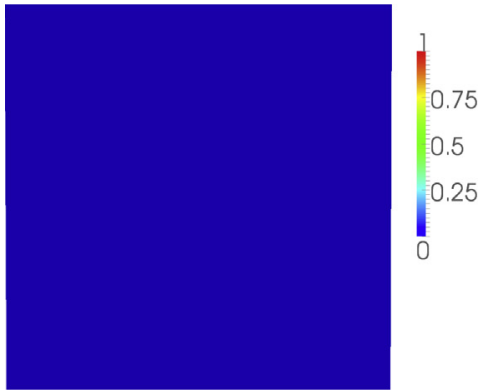

(a) $\xi=0$

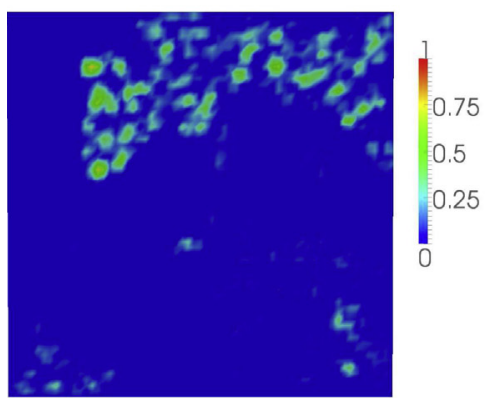

(c) $\xi=0.6846$

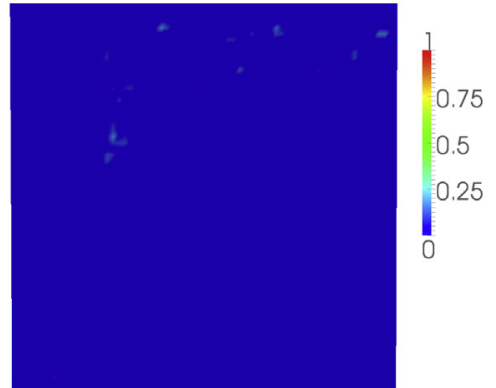

(b) $\xi=0.3375$

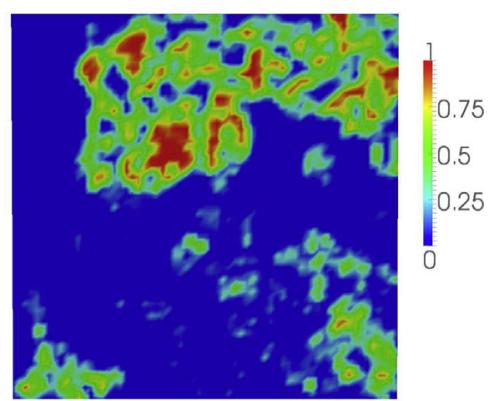

(d) $\xi=0.99$

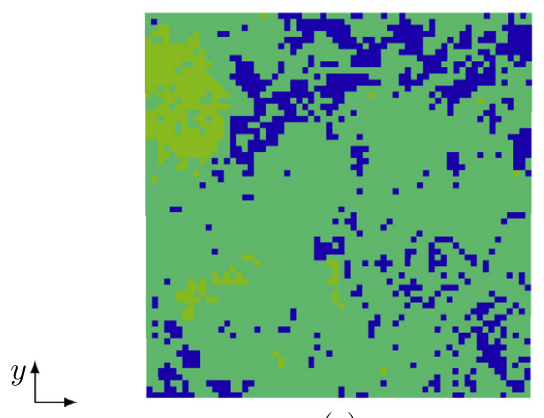

(e)
Hydration Product

Micropore

Unhydrated Residual

e)

Fig. 8. (a)-(d) damage distribution on a cross-section of HCP with various values of the chemical extent $\xi$ and (e) material distribution on the cross-section. 


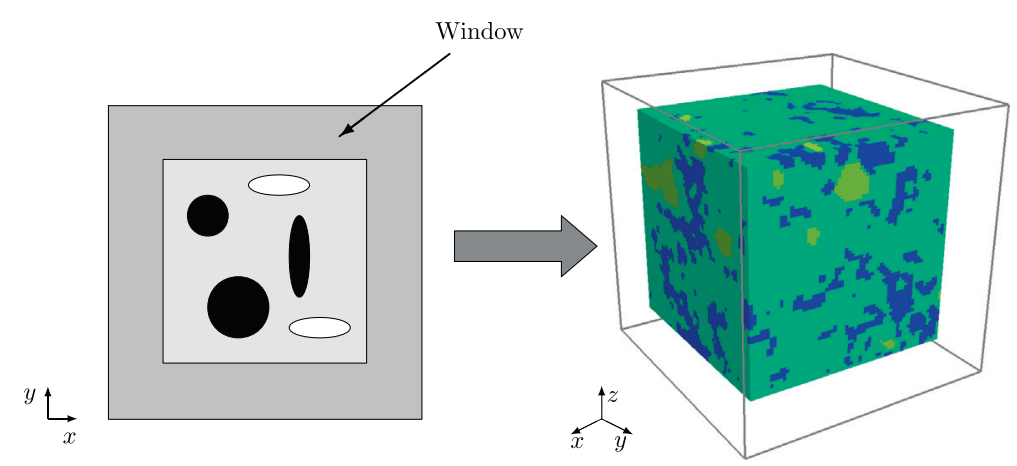

Fig. 9. Two-and three-dimensional representations of an HCP sample from a micro-CT scan, with 64 voxels per direction, embedded in a window.

homogeneous medium with a certain thickness, see Fig. 9, and an initial choice for its property is made. Linear displacement BCs are directly prescribed on the window. One then obtains a new effective property of the RVE, which is assigned to the window in a new iteration step. These iterations are repeated until the change of the effective property from one step to the next one is sufficiently small, thereby satisfying the condition of self-consistency. The window width of $4 \mu \mathrm{m}$ is adopted, see [50]. Principle stresses in an RVE with and without the window frame are shown, respectively, in Fig. 10.

Once the size of the RVE and the width of the window are determined, computational homogenization is carried out to obtain the effective chemical damage $D^{c}$. It is determined through the volume average of the isotropic damage variable defined in Section 3.2, which is triggered by the expansion of the gel. It is computed by imposing a zero average strain on the framed sample. The expansion coefficient of the gel at the microscale is still unknown, so that some reasonable values are tested to demonstrate the effective damage with respect to days, see Fig. 11, where the adopted correlation between chemical extent and days originates from Fig. 1. Note that the accumulated plastic strain in the RVE due to gel expansion is much smaller than the total strain, see Fig. 11. Accordingly, it is reasonable to assume that the microscale plasticity mechanisms do not significantly affect the mesoscale mechanical damage. This conclusion is expected since the chemical damage is predominantly due to gel expansion, which induces mostly volumetric strain and stress throughout the HCP. Fig. 12 display a comparison of the average volumetric and deviatoric stresses in

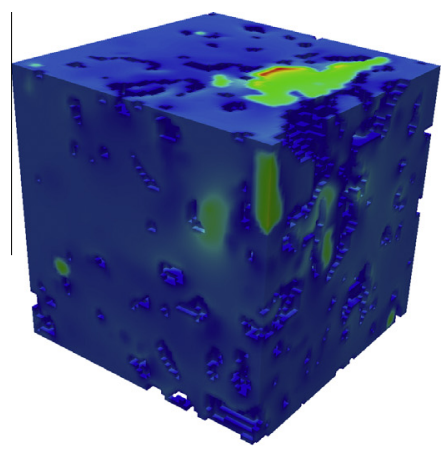

(a)
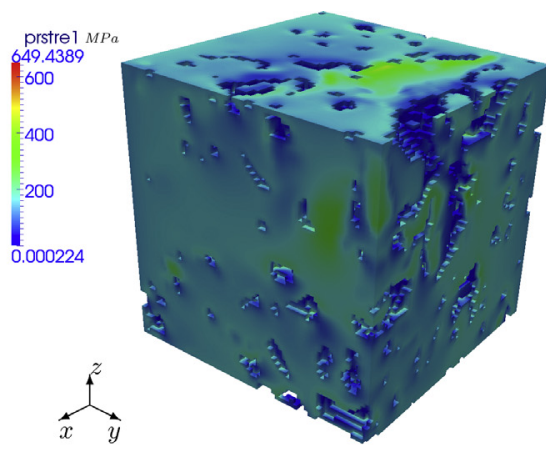

(b)

Fig. 10. (a) without the window method (micropores are not shown), and (b) with the window method (micropores and window are not shown).

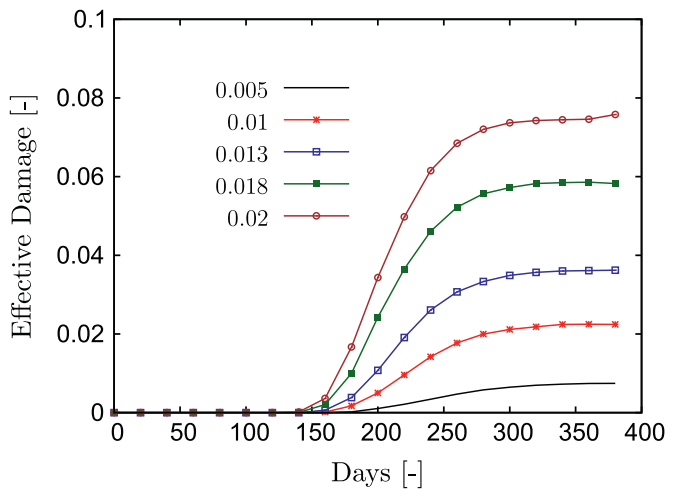

(a)

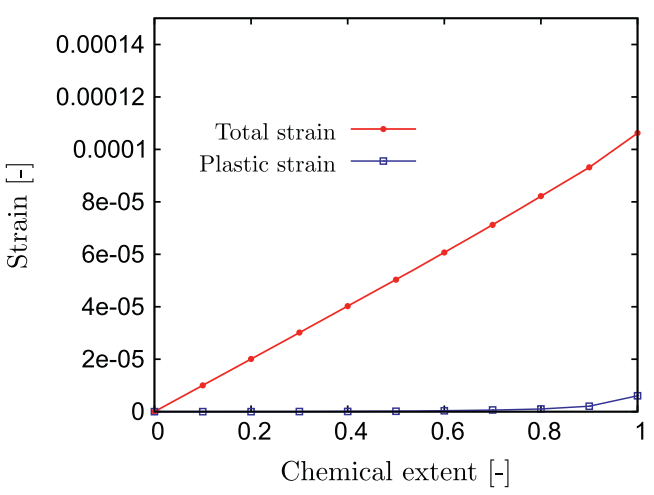

(b)

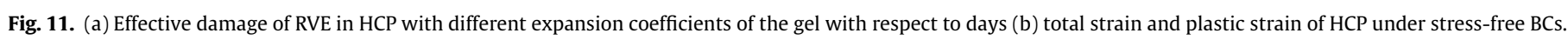




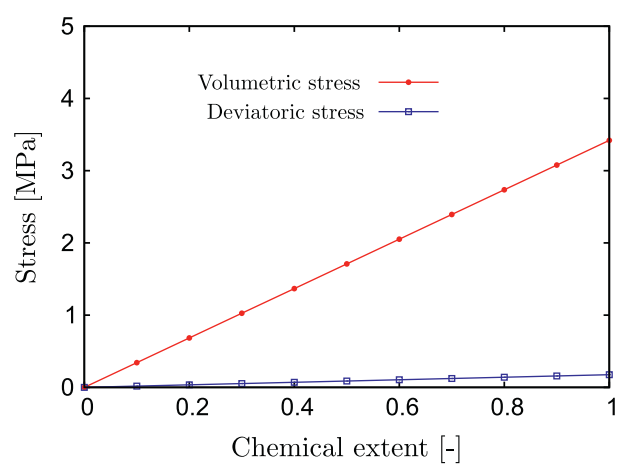

(a)

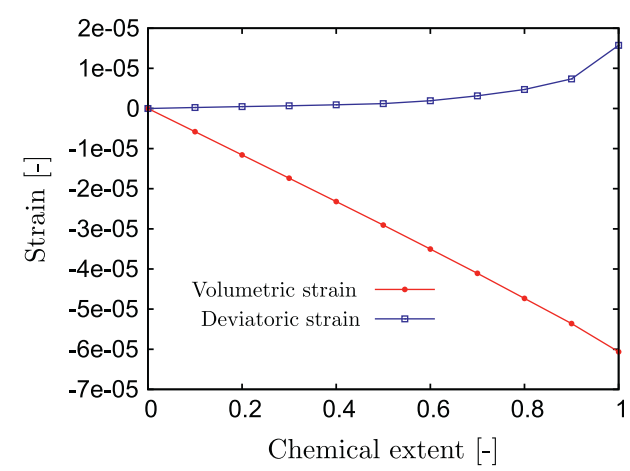

(b)

Fig. 12. (a) Average volumetric and deviatoric stresses under strain-free BCs, and (b) average volumetric and deviatoric strains under stress-free BCs on HCP.

the microstructure under strain-free BCs as well as a comparison of the average volumetric and deviatoric strains under stress-free BCs. The deviatoric portions are responsible for plasticity and they are seen to be much smaller than the volumetric ones. With the ability to upscale the ASR induced damage, the overall homogenized mesoscale response is sought next.

\section{Mesoscale of concrete}

Concrete at the mesoscale includes a binding matrix, aggregates and pores with a broad size distribution in addition to an interfacial zone between the aggregates and the matrix, an aspect that is omitted in the present study. The size, type, random distribution and physical property of the aggregates determine the performance of the concrete structure.

\subsection{Representation of mesoscale}

Several approaches exist for generating a realistic distribution of aggregates within the homogenized HCP matrix, such as the take-and-place and divide-and-place methods, see [57,58] for examples. In the present work, the take-and-place method is employed to generate the three-dimensional geometrical representation of concrete at the mesoscale. The size of the aggregates is obtained from the Fuller curve which is a certain aggregate size distribution yielding the optimal density and resembles the original concrete itself [59], where fine aggregates are not taken into account in this work [59]. A sample distribution with the size of $100 \times 100 \times 100 \mathrm{~mm}^{3}$ is shown in Fig. 13, where the volume fraction of the aggregates is $40 \%$. The resulting mesostructure is discretized by tetrahedral elements so that the mesh boundaries are coincident with the material interfaces, see Fig. 13.

\subsection{Effective inelastic constitutive equation}

Aggregates are assumed to be elastic in the present work. A visco-plastic model of the classical Perzyna-type combined with an isotropic damage, where symmetric damage surface is defined for tension and compression behaviors as a simplified case, is employed to represent the homogenized response of HCP. In order to capture the experimentally nonlinear behavior of concrete, the damage behavior is modeled through a damage surface $N$

$N\left(\epsilon^{e l}\right):=1-\exp \left[-\left(\frac{\epsilon^{e q}-a}{b}\right)^{c}\right]-D^{u} \leqq 0$

Here, $\boldsymbol{\epsilon}^{e q}$ is the elastic energy, $D^{u}$ denotes the mechanical damage, and $a, b$, and $c$ are material properties. In terms of the visco-plastic model, a penalty formulation $P$ is obtained from the stationarity of the dissipation

$P=-\boldsymbol{\sigma}: \dot{\epsilon^{p l}}+\frac{1}{\eta} \phi(f) \rightarrow$ stationary

subject to

$\phi(f)= \begin{cases}0 ; & <0 \\ \frac{1}{m+1} f^{m+1} ; & >0\end{cases}$

where the scalar viscosity $\eta$ represents a penalty factor and $\phi(f)$ denotes the penalty function. $m=1$ enables a nonlinear viscous behavior. The yield surface $f$ is described by the von Mises model

$f:=\|\operatorname{dev} \boldsymbol{\sigma}\|-\sqrt{\frac{2}{3}} k_{f}<0$

where $\operatorname{dev} \boldsymbol{\sigma}$ is the deviatoric part of the stress tensor and $k_{f}$ is the yield strength. The values $\left[k_{f}, \eta, \Delta t, b, a\right]=[20,1023,0.02$, 4241,718 ] reported in [2] captured the homogenized mechanical response of HCP sufficiently accurately.

\section{Two-step homogenization framework for parameter identification}

The chemically unstable characteristics of the gel poses challenges in experimentally measuring its mechanical properties. Alternatively, $\mathrm{FE}^{2}$-based homogenization can be utilized blackto obtain parameters at the microscale which are not easily measured through experiment, see [60] for a recent example. In the context of $\mathrm{FE}^{2}$, a coupled micro-macro simulation is carried out without the need for formulating an explicit homogenized formulation. If the resulting macroscopic mechanical behavior of the structure coincides with the experimental data, the parameters chosen at the microscale are deemed satisfactory $[60,48]$. In the present study, this method is not applicable due to its prohibitive computational cost. Instead, a two-step homogenization procedure is applied in a reverse manner based on the explicit homogenized mechanical response of HCP, with the goal of obtaining the expansion coefficient of the gel at the microscale, see Fig. 14.

\subsection{First step homogenization: HCP expansion strain determination}

Experimentally, it is observed that the dimensions of a concrete specimen suffering from ASR increase due to the swelling of the constituents [61]. The ASR induced volumetric expansion strain of the concrete specimen under stress-free conditions with respect to days is illustrated in Figs. 14 and 15. The hypothesis of the swell- 


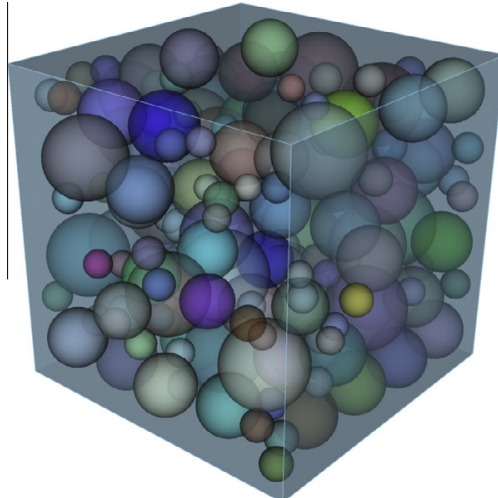

(a)

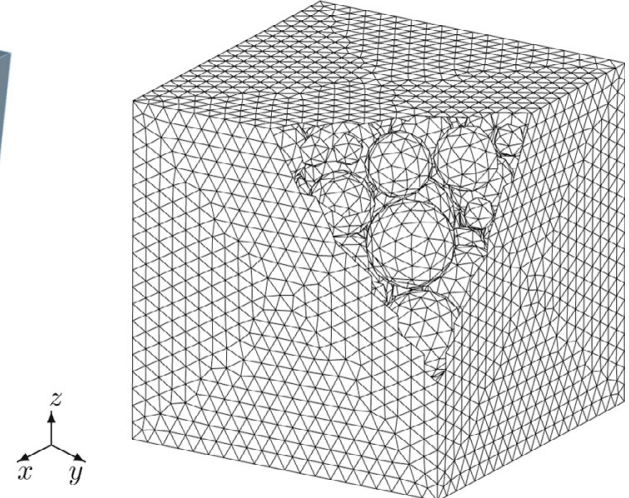

(b)

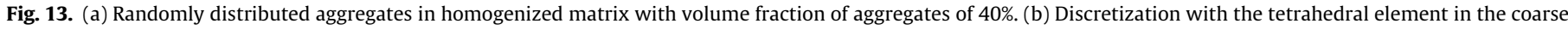
mesh.

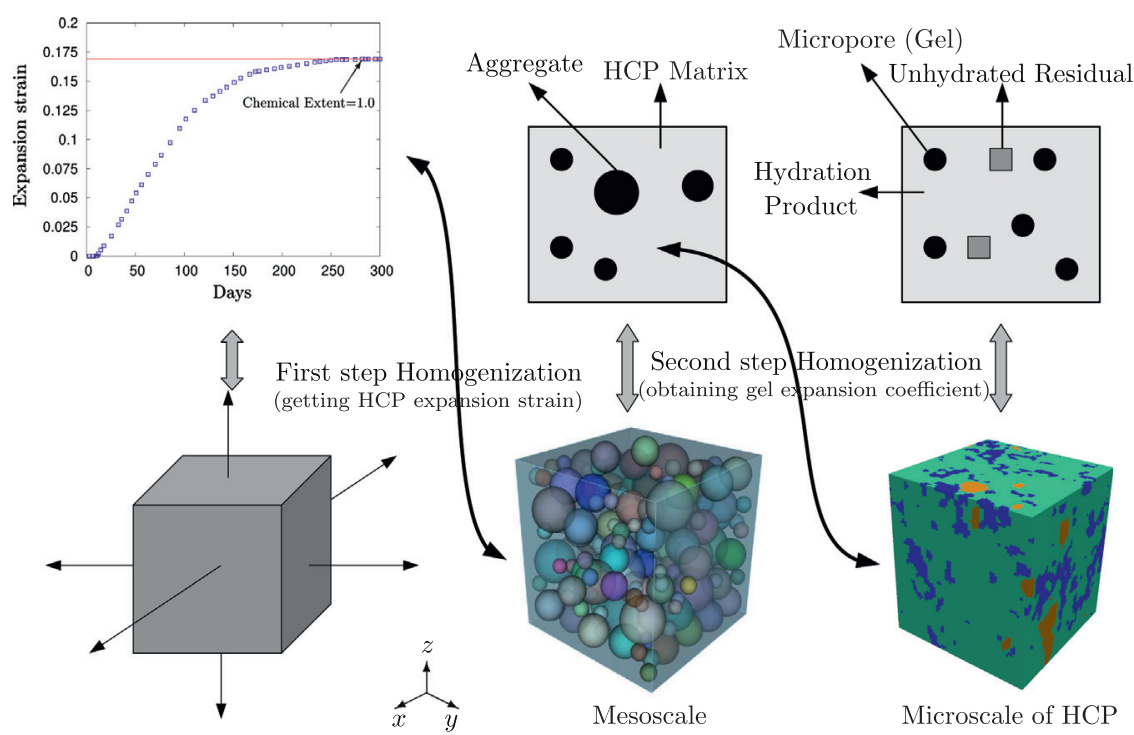

Fig. 14. Framework of two-step homogenization for parameter identification: link between concrete and HCP, and link between HCP and micropores.

ing mechanism in ASR employed in this work is that all gels are evenly produced and swell at the micropores of HCP. Consequently, the ASR induced expansion occurs in the HCP matrix of the mesoscale, rather than in the aggregates, thereby contributing to the macroscopic expansion of the concrete. In the context of numerical implementation, an additional term for the expansion strain due to ASR is incorporated into the inelastic constitutive equation of HCP described in Section 4.2, thus presenting that the concrete is suffering from ASR internally:

$\boldsymbol{\sigma}=\left(1-D^{\boldsymbol{u}}\right) E\left(\boldsymbol{\epsilon}-\boldsymbol{\epsilon}^{p l}-M \mathbf{1}\right)$

where $M$ denotes the ASR induced expansion strain in the HCP. The objective of the first step homogenization is to determine the value of $M$, so that the effective volumetric expansion strain of the concrete through computational homogenization approach only triggered by the expansion mechanism of ASR can coincide with the experimental data in Fig. 15. This is the classical way to determine parameters, which cannot be directly measured through experiment. The experiment from Fig. 15 was carried out under stress-free conditions. Presently, the stress-free state is generated through strain-control to prescribe BCs for

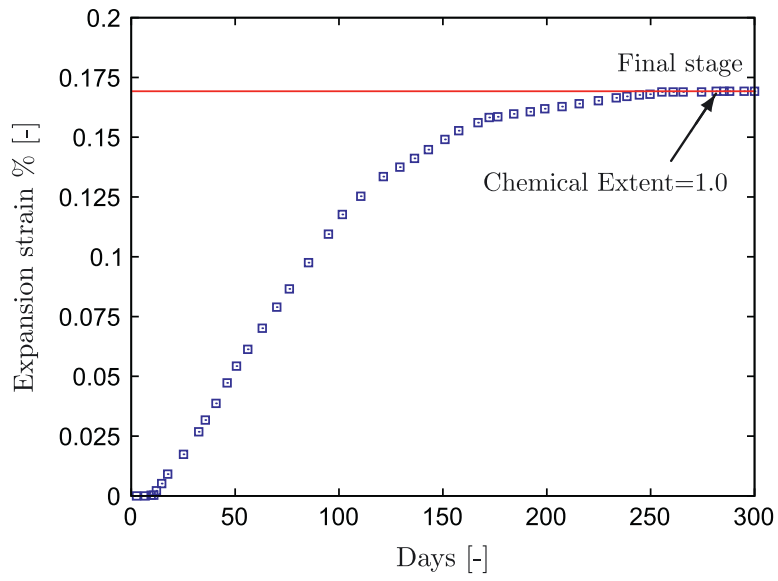

Fig. 15. Volumetric strain due to ASR in the concrete specimen under stress-free condition [61].

homogenization. The hypothesis that the gel retains permanent characteristics throughout the process renders it feasible to only 
Table 4

Algorithm of two-step homogenization to obtain the expansion coefficient of the gel at the microscale.

1. First step homogenization: The extra term $M$ as the expansion strain of HCP due to ASR is incorporated into the nonlinear constitutive equation of HCP at the mesoscale, see Section 4.2 .

a. Initial Step: $\boldsymbol{\sigma}=\left(1-D^{u}\right) E\left(\boldsymbol{\epsilon}-\boldsymbol{\epsilon}^{p l}-M \mathbf{1}\right)$.

b. Other steps: $\boldsymbol{\sigma}=\left(1-D^{u}-D^{c}\right) E\left(\boldsymbol{\epsilon}-\boldsymbol{\epsilon}^{p l}-M \mathbf{1}\right)$.

Run homogenization at the mesoscale, where $\boldsymbol{H}$ from Fig. 15 is adopted to impose linear displacement BCs. The expansion strain $M$ of HCP at the end of the reaction is obtained until the hydrostatic of the concrete equals to 0 .

2. Second step homogenization: Run homogenization in the RVE of HCP, where the obtained $M$ from the first step homogenization is adopted to prescribe displacement BCs. The expansion strain of the gel $A$ is acquired until the hydrostatic stress of HCP at the end of reaction is equal to 0 . Thus the expansion coefficient of the gel $\beta$ is obtained through $A=\beta \xi$, since $\xi$ equals to 1 .

3. Upscale damage: Run homogenization with the obtained $\beta$ in the RVE of HCP under strain-free BCs to calculate the effective ASR induced damage $D^{c}$ of HCP with respect to extent.

4. Check. Apply the sum of the damage from mechanical loading and chemical part to the constitutive equation of HCP at the mesoscale and run step 1 again.

a. If the change of $M^{\text {old }}$ and $M^{\text {new }}$ is small enough, stop computation.

b. If not, implement the step 2 again to get the new $\beta$. Then carry out the step 3 and step 4 again, until the change of $M$ from one step to the next step is small enough.

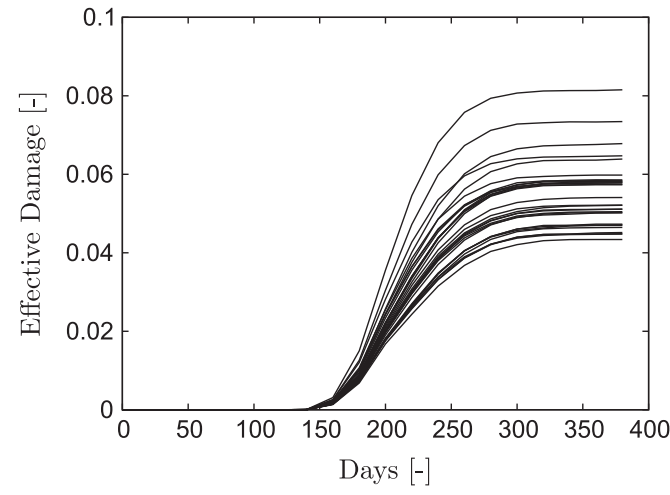

(a)

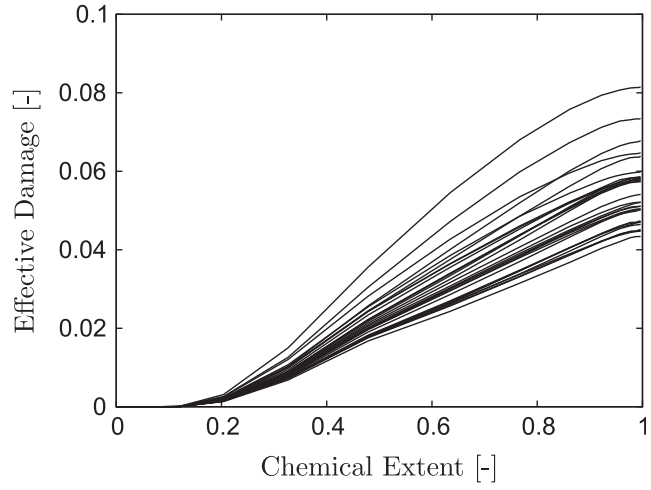

(b)

Fig. 16. Effective damage with respect to (a) days and (b) extent $\xi$, for 30 statistical tests.

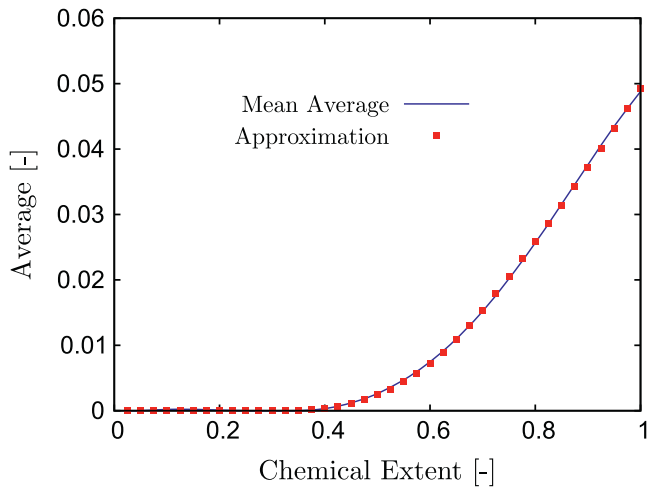

(a)

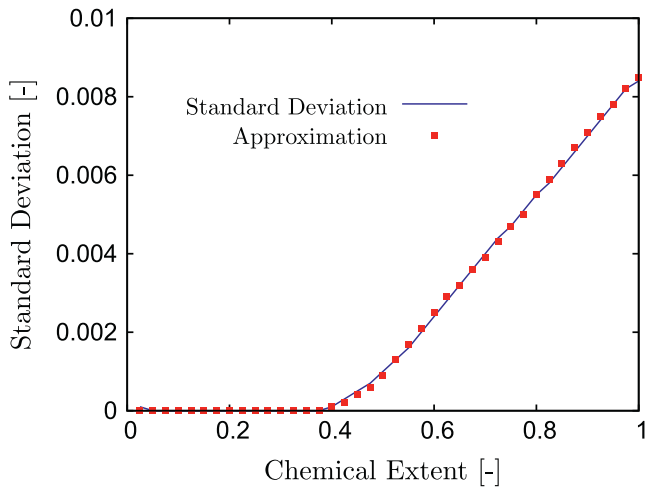

(b)

Fig. 17. Effective damage (a) mean and (b) standard deviation, for 30 statistical tests.

concentrate on fitting the final expansion strain as a simplified case, see the red line in Fig. 15, where the chemical extent $\xi$ is considered to be 1 . The constant strain tensor $\boldsymbol{H}$ defined by final volumetric expansion strain in Fig. 15 is applied to impose displacement BCs for homogenization through $\boldsymbol{u}=\boldsymbol{H} \cdot \boldsymbol{x}$ on $\partial \Omega$ [55], where $\boldsymbol{x}$ is the coordinates of nodes on $\partial \Omega$. $M$ is sought, until the hydrostatic stress of the concrete equals to zero at the end of the reaction. This method is also adopted in the second step of homogenization.

\subsection{Second step homogenization: gel expansion coefficient determination}

The second step of homogenization is to downscale to the microscale of HCP for determining the expansion coefficient of the gel. In this work, it is assumed that the swelling of the gel occurs at the micropores of $\mathrm{HCP}$, which results in the expansion of the HCP matrix and therefore of the concrete. The expansion strain of the gel is modeled as proportional to the chemical extent 
Table 5

Approximation coefficients of mean value and standard deviation of effective damage of statistical tests with respect to chemical extent.

\begin{tabular}{lrr}
\hline $\mathrm{i}$ & \multicolumn{1}{c}{$e_{i}^{\text {med }}$} & \multicolumn{1}{c}{$e_{i}^{\text {std }}$} \\
\hline 0 & 0.0809 & 0.0344 \\
1 & -0.1737 & -0.0581 \\
2 & 0.1284 & 0.0295 \\
3 & -0.0016 & -0.0002 \\
\hline
\end{tabular}

Table 6

Diffusivity properties of components at the mesoscale of concrete.

\begin{tabular}{ll}
\hline Components & Diffusivity $\mathrm{m}^{2} / \mathrm{s}$ \\
\hline HCP & $10^{-10}[62]$ \\
Aggregate & $1.85 \times 10^{-12}[63]$ \\
\hline
\end{tabular}

Table 7

Thermal properties of components at the mesoscale of concrete.

\begin{tabular}{lll}
\hline Property & Value (HCP) & Value (Aggregate) \\
\hline Capacity of heat $c$ & $1000 \mathrm{~J} / \mathrm{kg} \mathrm{K}[49]$ & $2600 \mathrm{~J} / \mathrm{kg} \mathrm{K}[64]$ \\
Heat conductivity $k$ & $1.4 \mathrm{~W} / \mathrm{mK}[49]$ & $2.828 \mathrm{~W} / \mathrm{mK}[46]$ \\
Density $\rho$ & $2120 \mathrm{~kg} / \mathrm{m}^{3}[49]$ & $2600 \mathrm{~kg} / \mathrm{m}^{3}[65]$ \\
\hline
\end{tabular}

$\xi: A=\beta \xi$, where $A$ is the expansion strain of the gel, and $\beta$ is the microscale expansion coefficient of the gel, independent of time according to earlier assumptions. The homogenization procedure explained in Section 5.1 is applied on the RVE of HCP with a window frame, where the $M$ obtained from the first step homogenization prescribes displacement $\mathrm{BCs}$ for homogenization, thus inducing stress-free conditions. This step also concentrates on the end of the reaction, where the chemical extent is equal to 1 . $\beta=0.0025$ is found to deliver negligible hydrostatic stress at the end of the reaction. Performing stress-free BCs through strain-control BCs for homogenization at the micro and mesoscale keeps the consistency in comparison to the expansion experiment implemented under stress-free BCs in Fig. 15.

The deterioration due to ASR from the microscale is not taken into account at the first step homogenization, see Eq. (5.1). However, it possibly affects the mechanical property and the subsequent expansion behavior of the concrete specimen. Its effect is investigated as follows. The obtained expansion coefficient $\beta$ is adopted to calculate the effective damage of HCP due to ASR through homogenization approach with respect to extent, where strain-free BCs are prescribed. The chemical effective damage is then incorporated into the inelastic constitutive equation of HCP to run the first step of homogenization again. The next task is to evaluate whether the change of the old $M$ and the new $M$ can be neglected. If not, it is necessary to carry out the second step of homogenization with the new $M$ again. Iterations are carried out until the change of $M$ from one step to the next step is small enough. The algorithm is summarized in Table 4.

\subsection{Statistical analysis associated with homogenization}

The approximate RVE used in previous computations is only a randomly chosen sample from a much larger micro-CT scan of HCP. Hence, it is indispensable to carry out statistical tests to address the effect of randomness. For this purpose, 30 randomly obtained RVEs from a micro-CT scan are tested. The resulting effective damage due to the expansion of the gel with respect to days and chemical extent are shown in Fig. 16, respectively. The corresponding mean value and standard deviation of the effective damage are calculated as functions of the chemical extent, see red points in Fig. 17, via the polynomial expansions

$D^{c}(\xi)^{\text {med }}:=\sum_{i=0}^{i \leqslant 3} e_{i}^{\text {med }} \xi^{i} \quad$ and $\quad D^{c}(\xi)^{\text {std }}:=\sum_{i=0}^{i \leqslant 3} e_{i}^{\text {std }} \xi^{i}$.

The coefficients of approximation are listed in Table 5. This correlation between effective damage due to ASR and chemical extent can be directly upscaled to HCP at the mesoscale of the concrete. Since the chemical extent has been projected as a constant to the RVE, hydro-thermo-chemical coupling does not manifest itself at this scale. In order to reflect the observations that ASR is activated when the threshold of relative humidity is achieved, see Section 2.3, and that ASR induced damage occurs at the mesoscale after the chemical extent reaches to 0.3 , see Fig. 17, full coupling needs to be addressed at the mesoscale. This is the goal of the following section.

\section{Numerical coupling at mesoscale}

It is experimentally observed that the progress of ASR relies on the temperature and the relative humidity [41]. The associated influences have individually been discussed in Section 2.2 and 2.3. In this section, the mesoscopic local values of the transient temperature and relative humidity are obtained through the solution of the instationary thermal conduction and diffusion equations. Together, they locally determine the deterioration due to ASR at the microscale, based on the formulation obtained in the previous section.

The instationary diffusion equation states

$\dot{S}+\operatorname{div} \mathbf{J}=0$

where $\boldsymbol{J}=-d \nabla S$ from Fick's law is the diffusion flux, $S$ is the relative humidity and $d$ is the diffusivity. The diffusivity of components at the mesoscale are listed in Table 6.

On the other hand, the instationary thermal conduction is described by

$\rho c \dot{\theta}+\operatorname{div} \boldsymbol{q}=0$

where $\rho$ is the density, $c$ is the heat capacity, $\boldsymbol{q}=-K \nabla \theta$ from the Fourier law is the thermal flux, $\theta$ is the temperature, and $K$ is the

Table 8

Multiscale hydro-thermo-chemo-mechanical coupling in concrete based on staggering.

1. Diffusion field. Update the relative humidity $S^{n+1}$ through Eq. (6.1) at the mesoscale

2. Thermal field. Update the temperature $\theta^{n+1}$ through Eq. (6.2) at the mesoscale

3. Chemical extent. Use $S^{n+1}$ and $\theta^{n+1}$ to update the chemical extent to $\xi^{n+1}$, see Sections 2.2 and 2.3

4. Link meso \& micro scales. Use the obtained chemical extent $\xi^{n+1}$ at the mesoscale to determine the ASR induced damage at the microscale, through the correlation between chemical extent and damage due to ASR, see Eq. (5.2)

5. Mechanical field. Apply the sum of the mechanical damage $D^{u}$ and chemical damage $D^{c}$

$$
D=D^{u}+D^{c}
$$

to the nonlinear constitutive law of HCP in order to obtain $u^{n+1}$

6. Increase time step. Update all the field variables and set the time step forward to go back to step 1 


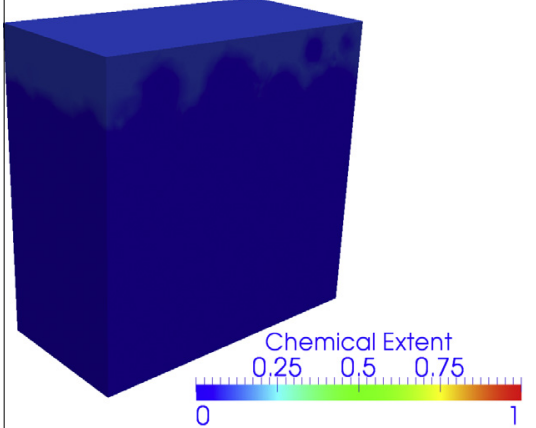

(a)

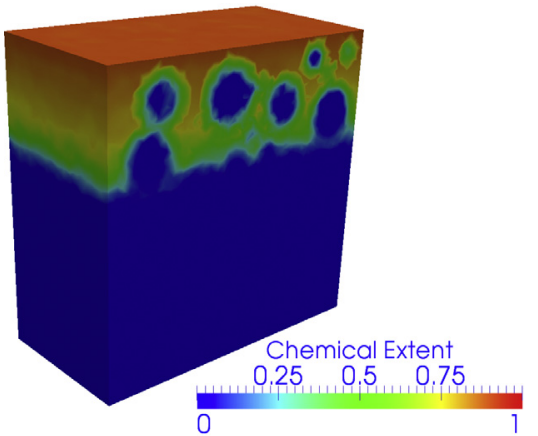

(c)

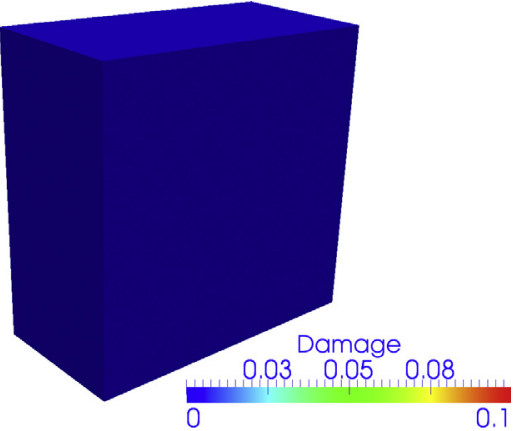

(b)

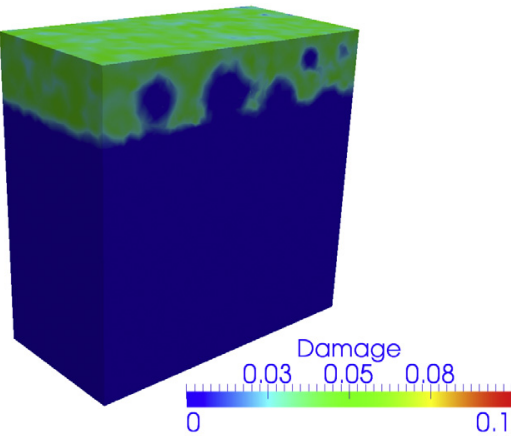

(d)

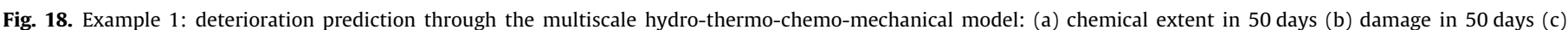
chemical extent in 300 days (d) damage in 300 days.

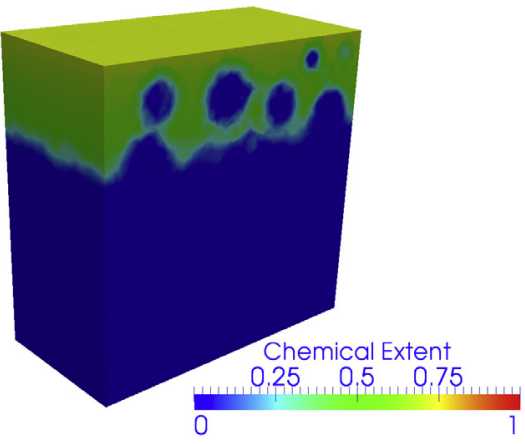

(a)

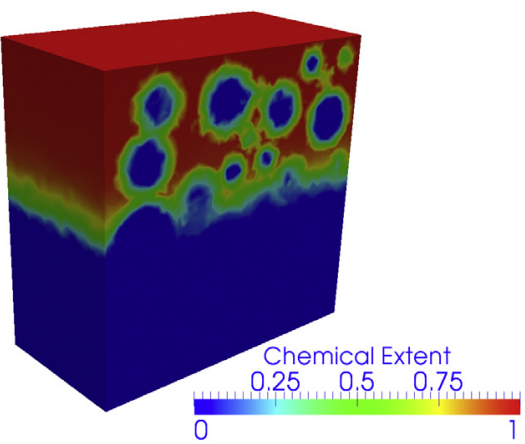

(c)

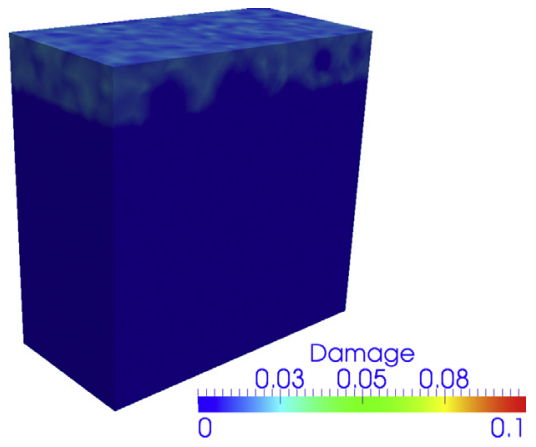

(b)

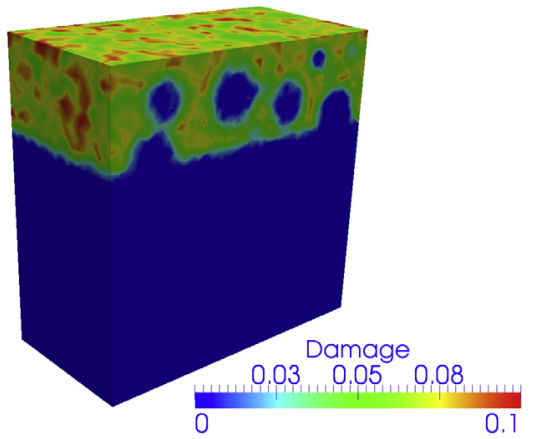

(d)

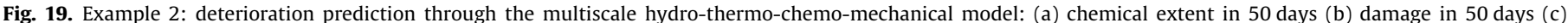
chemical extent in 300 days (d) damage in 300 days.

thermal conductivity. The thermal properties of the components at the mesoscale are described in Table 7.
In order to solve the coupled problem, a staggered approach is adopted, see $[66,67]$. Staggering usually has the disadvantage of a 
small time step restriction in view of its explicit nature. However, it has a significantly simpler algorithmic structure compared to an implicit scheme due to the sequential solution of the coupled system of field equations. Once the transient temperature and relative humidity are obtained through instationary thermal conduction and diffusion equations at the mesoscale of the concrete, the chemical extent $\xi$ at the material point of mesoscale can be updated. The obtained chemical extent can directly determine the damage induced by ASR at the microscale through the correlation between effective damage of HCP and chemical extent. In other words, staggering is employed at the mesoscale of the concrete to solve the coupled problem, yet it upscales the chemical damage quantity from the microscale during the process. Supposing that the equilibrium states for diffusion, conduction and mechanical problems have been obtained at time $t_{n}$, the procedure for obtaining the solutions at time $t_{n+1}=t_{n}+\delta t$ is described in Table 8 .

Two simple examples are tested in this section. The first example enforces the varying temperature and relative humidity BCs with respect to time on the top surface of the mesoscale representation of the concrete, see curve b in Fig. 2 and curve b in Fig. 3. The multiscale simulation results predict the deterioration due to ASR as shown in Fig. 18. In the second example, constant relative humidity 1.0 and temperature $20^{\circ} \mathrm{C}$ are prescribed on the top surface of the mesoscale representation of the concrete. One can obtain the evolution of chemical extent and resulting damage in Fig. 19.

Two examples present that ASR is a relative show reaction and it only occurs in HCP. The evolution of the ASR induced damage follows the extent of reaction. In other words, the chemical extent of ASR determines in which region damage occurs more easily in the concrete. Since the initial temperature and relative humidity are not taken into account in the concrete, temperature and relative humidity mediated chemical extent evolves from upper to lower surface of the concrete. Different evolution performances of chemical extent and induced damage as shown in Fig. 18 and 19 can be explained by the contributions of constant and varying temperature and relative humidity, see Sections 2.2 and 2.3.

\section{Conclusion}

Problems associated with the Alkali-Silica Reaction (ASR) in concrete structures usually arise several years after construction. The objective of the present work was to establish a multiscale model to predict the deterioration due to ASR in concrete with the goal of accelerating the prediction of the extent of damage in comparison to experimental procedures. The calculations were based on 3D micro-CT scans of the microscale hardened cement paste (HCP) and randomly generated mesoscale aggregate distributions at one higher scale. Starting from the assumption that the gels are evenly produced in the micropores of HCP and exert uniform pressure on the surrounding material, the expansion coefficient of the gel at the microscale was obtained through a twostep homogenization approach. Based on a correlation between the effective damage and the chemical extent, the simulation of ASR induced deterioration at the mesoscale was carried out through a coupled hydro-thermo-chemo-mechanical framework in a staggered setting.

The present work concentrated on the establishment of a multiscale framework to predict the failure induced by ASR at the lower scale. Since ASR is an extremely complex reaction in concrete and only one of various possible mechanisms has been adopted in this work, the multiscale model is limited in its ability to comprehensively predict the failure caused by ASR. For this reason, a comparison between the numerical simulations and experimental data has not been attempted. However, the authors are planning to model the localization of the ASR induced failure at the interfacial zone between the aggregates and HCP, as well as the crack through aggregates, in order to improve the reliability of the advocated framework to analyze ASR $[15,68]$. Additionally, the symmetric damage surface employed in this work cannot correctly describe the different behavior of HCP in tension/compression. The implementation of improved models is planned as future work Com3. Nevertheless, despite its present lack of predictive capability, the possibility to extend this multiscale approach towards additional ASR mechanisms, as well as to other closely related phenomena such as Delayed Ettringite Formation (DEF) [69] or Alkali-Carbonate Reaction (ACR) [70], highlights its significance.

\section{Acknowledgement}

The authors would like to thank Stéphane Multon in Toulouse University and Kefei Li in Tsinghua University for helpful discussions concerning the mechanism of alkali-silica reaction.

\section{References}

[1] V.P. Nguyen, M. Stroeven, L.J. Sluys, Eng. Fract. Mech. 201-204 (2012) 139156.

[2] M. Hain, P. Wriggers, Finite Elem. Anal. Des, 44 (2008) 233-244.

[3] F.J. Ulm, O. Coussy, K.F. Li, C. Larive, J. Eng. Mech. 126 (2000) 233-242.

[4] E. Denarié, C. Cécot, C. Huet, Cem. Concr. Res. 36 (2006) 571-575.

[5] G. Cusatis, L. Cedolin, Eng. Fract. Mech. 74 (2007) 3-17.

[6] S. Eckardt, C. Könke, J. Algor. Comput. Technol. 2 (2008) 275-297.

[7] A. Ghosh, P. Chaudhuri, Comput. Mater. Sci. 69 (2013) 204-215.

[8] A.E. Idiart, C.M. López, I. Carol, Cem. Concr. Compos. 33 (2011) 411-423.

[9] E. Lemarchand, L. Dormieux, F.J. Ulm, Philos. Trans. R. Soc. Math., Phys., Eng. Sci. 363 (2005) 2581-2602.

[10] Z.P. Bažant, A. Steffens, Cem. Concr. Res. 30 (2000) 419-428.

[11] J.K. McGowan, H.E. Vivian, Aus. J. Appl. Sci. 3 (1952) 228-232.

[12] G.M. Idorn, Cem. Concr. Res. 31 (2001) 1109-1110.

[13] E. Garcia-Diaz, J. Riche, D. Bulteel, C. Vernet, Cem. Concr. Res. 36 (2006) 395 400.

[14] T. Ichikawa, M. Miura, Cem. Concr. Res. 37 (2007) 1291-1297.

[15] S. Multon, A. Sellier, M. Cry, Cem. Concr. Res. 39 (2009) 490-500.

[16] C.F. Dunant, K.L. Scrivener, Cem. Concr. Res. 40 (2010) 517-525.

[17] M.B. Haha, E. Gallucci, A. Guidoum, K.L. Scrivener, Cem. Concr. Res. 37 (2007) $1206-1214$.

[18] J.M. Ponce, O.R. Batic, Cem. Concr. Res. 36 (2006) 1148-1156.

[19] R.G. Charlwood, Int. J. Hydro. Dams 10 (1994) 73-80.

[20] G.A. Thompson, R.G. Charlwood, R.R. Steele, D.D. Curtis, Mactaquac generating station intake and spillway remedial measures, in: Proceedings for 18th International Congress on Large Dams, vol. 1, Q-68, R.24, Durban, South Africa, 1994, pp. 73-80.

[21] B. Capra, A. Sellier, Mech. Mater. 35 (2003) 817-830.

[22] S. Pietruszczak, Comput. Struct. 58 (1996) 1093-1097.

[23] M.F. Herrador, F. Martínez-Abella, R.D.H. Fernández-Gago, Mater. Struct. 42 (2009) 201-212.

[24] V. Saouma, L. Perotti, ACI Mater. J. 103 (2006) 194-202.

[25] P. Leger, P. Coté, R. Tinawi, Comput. Struct. 60 (1996) 601-611.

[26] M.C.R. Farage, J.L.D. Alves, E.M.R. Fairbairn, Cem. Concr. Res. 34 (2004) 495 505.

[27] E.M.R. Fairbairn, F.L.B. Ribeiro, L.E. Lopes, R.D. Toledo-Filho, M.M. Silvoso, Commun. Numer. Meth. Eng. 22 (2006) 1-12.

[28] M. Huang, S. Pietruszczak, Mech. Cohes.-Frict. Mater. 1 (1996) 305-319.

[29] C. Comi, R. Fedele, U. Perego, Mech. Mater. 41 (2009) 210-230.

[30] F. Bangert, D. Kuhl, G. Meschke, Int. J. Numer. Anal. Meth. Geomech. 28 (2004) 689-714.

[31] C. Comi, B. Kirchmayr, R. Pignatelli, Int. J. Solids Struct. 49 (2012) 3367-3380.

[32] F. Pesavento, D. Gawin, M. Wyrzykowski, B.A. Schrefler, L. Simoni, Comput. Meth. Appl. Mech. Eng. 225-28 (2012) 95-115.

[33] J.H. Shin, Modeling Alkali-Silica Reaction Using Image Analysis and Finite Element Method, Ph.D. thesis, University of Illinois at Urbana-Champaign, Urbana-Champaign, USA, 2009.

[34] C. Dunant, Experimental and Modelling Study of the Alkali-Silica-Reaction in Concrete, Ph.D. thesis, Ecole Polytechnique Fédérale de Lausanne, Lausanne, Switzerland, 2009.

[35] Z.P. Bažant, C. Meyer, J. Eng. Mech. 126 (2000) 226-232.

[36] L. Charpin, A. Ehrlacher, Cem. Concr. Res. 42 (2012) 613-625.

[37] I. Comby-Peyrot, F. Bernard, P.O. Bouchard, F. Bay, E. Garcia-Diaz, Comput Mater. Sci. 46 (2009) 1163-1177.

[38] M. Alnaggar, G. Cusatis, G.D. Luzio, Cem. Concr. Compos. 41 (2013) 45-59.

[39] J.W. Pan, Y.T. Feng, J.T. Wang, Q.C. Sun, C.H. Zhang, D.R.J. Owen, Front. Struct. Civil Eng. 6 (2012) 1-18.

[40] R. Dron, F. Brivot, Cem. Concr. Res. 23 (1993) 93-103. 
[41] C. Larive, Apports combinés de l'expérimentation et de la modélisation à la compréhension de l'alcali-réaction et de ses effects mécaniques, Ph.D. thesis, Laboratoire Central des Ponts et Chaussées, Paris, France, 1998.

[42] T. Kurihara, K. Katawaki, Effects of moisture control and inhibition on alkali silica reaction, in: Proceedings for 8th International Conference on Alkali Aggregate Reaction in Concrete, Kyoto, Japan, 1989, pp. 629-634.

[43] L.O. Nilsson, Moisture effects on the alkali-silica reaction, in: Proceedings for 6th International Conference on Alkali in Concrete, Copenhagen, 1989. pp. 201-208.

[44] F. Tomosawa, K. Tamura, M. Abe, Influence of water content of concrete on alkali-aggregate reaction, in: Proceedings for 8th International Conference on Alkali Aggregate Reaction in Concrete, Kyoto, Japan, 1989, pp. 881-885.

[45] U. Ludwig, Effects of environmental conditions on alkali-aggregate reaction and preventive measures, in: Proceedings for 8th International Conference on Alkali Aggregate Reaction in Concrete, Kyoto, Japan, 1989, pp. 583-596.

[46] T. Wu, İ. Temizer, P. Wriggers, Cem. Concr. Compos. 35 (2012) 59-70.

[47] İ. Temizer, P. Wriggers, J. Mech. Phys. Solids 59 (2011) 344-372.

[48] Q. Yu, J. Fish, Int. J. Solids Struct. 39 (2002) 6429-6452.

[49] M. Hain, Computational Homogenization of Micro-Structural Damage Due to Frost in Hardened Cement Paste, Ph.D. thesis, Leibniz Universität Hannover, Hannover, Germany, 2007.

[50] M. Hain, P. Wriggers, Comput. Mech. 42 (2008) 197-212.

[51] J.W. Phair, S.N. Tkachev, M.H. Manghnani, R.A. Livingston, J. Mater. Res. 20 (2005) 344-349.

[52] E.F.I. Boerner, P. Wriggers, Comput. Mech. 42 (2008) 407-416.

[53] D.S. Müller-Hoeppe, S. Löhnert, P. Wriggers, Int. J. Numer. Meth. Eng. 78 (2009) 1164-1187.

[54] P. Wriggers, Nonlinear Finite Element Methods, Springer, Berlin Heidelberg, 2008
[55] T.I. Zohdi, P. Wriggers, Introduction to Computational Micromechanics, Springer, Berlin, Heidelberg, New York, 2005.

[56] İ. Temizer, T. Wu, P. Wriggers, Int. J. Eng. Sci. 64 (2013) 66-73.

[57] S. Torquato, Random Heterogeneous Materials: Microstructure and Macroscopic Properties, Springer, Berlin, Heidelberg, New York, 2002.

[58] G. De. Schutter, L. Taerwe, Mater. Struct. 26 (1993) 67-73.

[59] P. Wriggers, S.O. Moftah, Finite Elem. Anal. Des. 42 (2006) 623-636.

[60] U. Schmidt, J. Mergheim, P. Steinmann, Int. J. Multiscale Comput. Eng. 10 (2012) 327-342.

[61] N. Smaoui, M.A. Bérubé, B. Fournier, B. Bissonnette, B. Durand, Can. J. Civil Eng. 31 (2004) 826-845.

[62] M.Z. Zhang, G. Ye, K.V. Breugel, Construct. Build. Mater. 25 (2010) 2046-2052.

[63] K.L. Vasconcelos, A. Bhasin, D.N. Little, R.L. Lytton, J. Mater. Civil Eng. 23 (2011) 445-452.

[64] U. Kodide, Thermal Conductivity and Its Effects on the Performance of Pcc Pavements in MEPDG, Ph.D. thesis, Louisiana State University, Baton Rouge, USA, 2010.

[65] American Concrete Institute Education Bulletin. Aggregates for concrete. Farmington Hills, MI: American Concrete Institute, E1-07, 1999.

[66] T.I. Zohdi, Comput. Meth. Appl. Mech. Eng. 193 (2004) 679-699.

[67] P. Erbts, A. Düster, Comput. Math. Appl. 64 (2012) 2408-2430.

[68] M.B. Haha, Mechanical Effects of Alkali Silica Reaction in Concrete Studied by SEM-Image Analysis, Ph.D. thesis, Ecole Polytechnique Fédérale de Lausanne, Lausanne, Switzerland, 2006.

[69] S. Diamond, Cem. Concr. Res. 30 (2000) 1987-1991.

[70] ZZ Xu, X.H. Lan, M. Deng M.S. Tang A new accelerated method for determining the potential alkali-carbonate reactivity, Cem. Concr. Res. 32 (2002) 851-857. 